2020/04/12/mortality-associated-with-covid-19outbreaks-in-care-homes-early-international-evidence

4. Etard J-F, Vanhems P, Atlani-Duault L, Ecochard R. Potential lethal outbreak of coronavirus disease (COVID-19) among the elderly in retirement homes and long-term facilities, France, March 2020. Euro Surveill. 2020;25:8-10. https:/ / doi.org/10.2807/1560-7917.ES.2020.25.15.2000448

5. Rada AG. Covid-19: the precarious position of Spain's nursing homes. BMJ. 2020;369:m1554. https://doi.org/ 10.1136/bmj.m1554

6. ECDC Public Health Emergency Team, Danis K, Fonteneau L, Georges S, Daniau C, Bernard Stoecklin S, et al. High impact of COVID-19 in long-term care facilities, suggestion for monitoring in the EU/EEA, May 2020. Euro Surveill. 2020;25:1-5. https:// doi.org/10.2807/1560-7917. ES.2020.25.22.2000956

7. Ministerio de la Presidencia relaciones con las cortes y memoria democrática. Royal Decree 463/2020 (March 14) declaring the alarm state for the health crisis management situation caused by COVID-19. BOE no 67 from March 14, 2020 [in Spanish]. 2020 Mar 14;67(I):25390-400 [cited 2020 May 21]. https://www.boe.es/diario_boe/txt.php?id= BOE-A-2020-3692

8. Ministerio de Sanidad. Guideline for the prevention and control of COVID-19 in nursing homes and other residential social services [in Spanish]. 2020 [cited 2020 May 21]. https://www.mscbs.gob.es/profesionales/ saludPublica/ccayes/alertasActual/nCov-China/ documentos/Residencias_y_centros_sociosanitarios_ COVID-19.pdf

9. Arons MM, Hatfield KM, Reddy SC, Kimball A, James A, Jacobs JR, et al.; Public Health-Seattle and King County and CDC COVID-19 Investigation Team. Presymptomatic SARS-CoV-2 infections and transmission in a skilled nursing facility. N Engl J Med. 2020;382:2081-90. https:/ / doi.org/10.1056/NEJMoa2008457

10. He X, Lau EHY, Wu P, Deng X, Wang J, Hao X, et al. Temporal dynamics in viral shedding and transmissibility of COVID-19. Nat Med. 2020;26:672-5. https:/ / doi.org/ $10.1038 / \mathrm{s} 41591-020-0869-5$

Address for correspondence: Magda Campins, Servei de Medicina Preventiva i Epidemiologia, Vall d'Hebron Hospital Universitari, Vall d'Hebron Barcelona Hospital Campus, Passeig Vall d'Hebron 119-129, 08035 Barcelona, Spain; email: mcampins@vhebron.net

\section{Leuconostoc lactis and Staphylococcus nepalensis Bacteremia, Japan}

\author{
Satoshi Hosoya, Satoshi Kutsuna, Daisuke Shiojiri, \\ Saeko Tamura, Erina Isaka, Yuji Wakimoto, \\ Hidetoshi Nomoto, Norio Ohmagari
}

Author affiliation: National Center for Global Health and Medicine, Tokyo, Japan

DOI: https://doi.org/10.3201/eid2609.191123

Leuconostoc lactis is a glycopeptide-resistant, grampositive, facultative anaerobic coccus isolated from dairy products, whereas Staphylococcus nepalensis is coagulase-negative coccus that has not been identified as human pathogen. We report an instructive case of $L$. lactis and $S$. nepalensis bacteremia in a 71-year-old man who experienced Boerhaave syndrome after a meal.

Teuconostoc lactis is an intrinsically glycopeptide-reListant but ampicillin-susceptible, gram-positive, facultative anaerobic coccus (1) found in food products including dairy products, vegetables, and wine. L. lactis is a very rare pathogen associated with bloodstream infections (2). Staphylococcus nepalensis is a novobiocin-resistant coagulase-negative staphylococcus also found in food products, such as dry-cured ham and fish sauce, that has not been reported as a human pathogen (3-5). Neither L. lactis nor S. nepalensis is part of normal human bacterial flora $(2,3)$.

A 71-year-old man with hypertension and hyperlipidemia sought care for upper abdominal pain and vomiting after a meal at his son's restaurant. A computed tomography (CT) scan showed collapse of the lower esophagus wall and expansion of the mediastinum; medical staff diagnosed a spontaneous esophageal rupture and performed emergency surgery. Surgical findings demonstrated a $5 \mathrm{~cm}$ perforation of the lower esophagus with no rupture to the thoracic and abdominal cavity. The final diagnosis included Boerhaave syndrome, esophageal hiatus hernia, and mediastinitis. Two sets of blood culture taken on day 1 were positive for gram-positive cocci, which we identified by matrix-assisted laser desorption/ionization time-of-flight (MALDI-TOF) mass spectrometry as L. lactis in an aerobic bottle $(10.7 \mathrm{~h}$ to culture) and an anaerobic bottle (13.3 h to culture) and S. nepalensis in 1 anaerobic bottle $(24.3 \mathrm{~h}$ to culture). The 2 bacteria were indications of true bacteremia; therefore, we escalated ampicillin/sulbactam (treatment to piperacillin/tazobactam for L. lactis (Appendix Table 1, https://wwwnc.cdc.gov/EID/ 


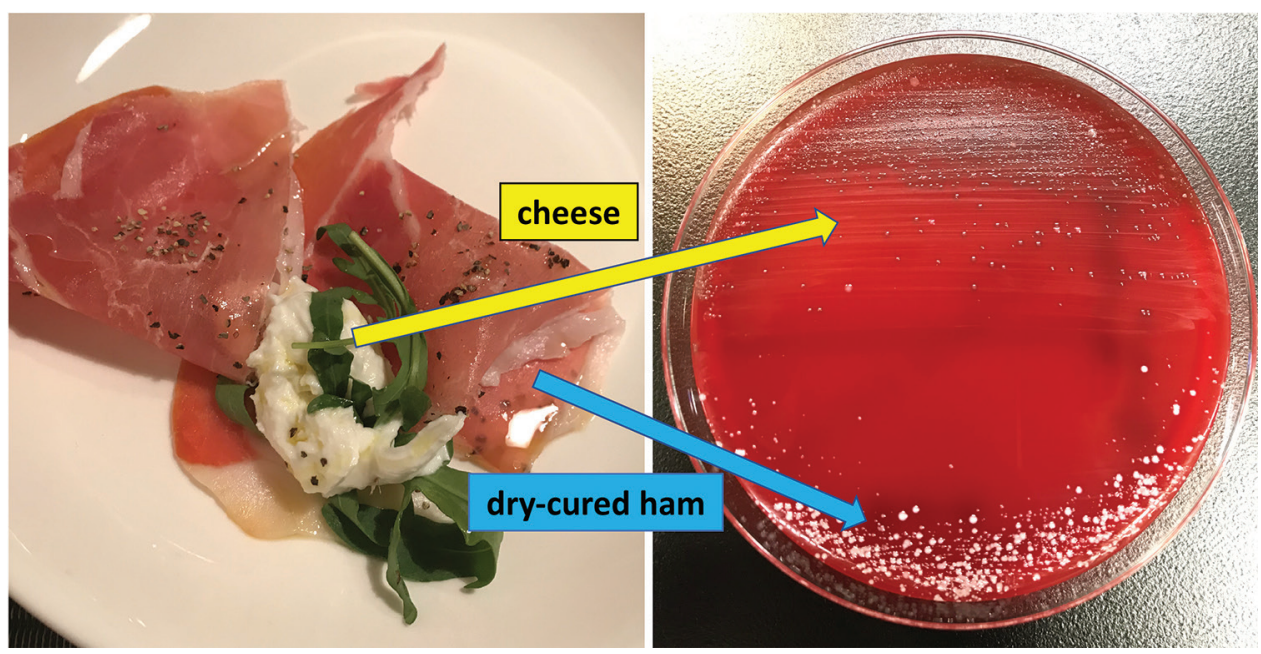

Figure. Culture of cheese and dry-cured ham on blood agar medium from investigation of patient with Leuconostoc lactis and Staphylococcus nepalensis bacteremia, Japan. The colonies, cultured from cheese, were identified as $L$. lactis by matrix-assisted laser desorption/ionization time-offlight mass spectrometry mass spectrometry; however, the colonies derived from dry-cured ham were identified as S. equorum and S. xylosus but not as S. nepalensis.

article/26/9/19-1123-App1.pdf) and initiated vancomycin treatment for $S$. nepalensis on day 3 after admission (Appendix Table 2). We measured MICs in the microdilution method using the MicroScan WalkAway 96 SI system (Beckman Coulter, https:/ / www. beckmancoulter.com) with a MICrofast7J panel and determined the susceptibility of L. lactis according to Clinical and Laboratory Standards Institute (CLSI) guidelines (6). On day 7, we deescalated piperacillin/tazobactam to ampicillin/sulbactam, referring to the MICs, and we obtained follow-up sets of blood culture. The culture results were negative. We discontinued vancomycin by day 14 but maintained the ampicillin/sulbactam regimen. A follow-up CT scan on day 28 showed a subsiding mediastinal abscess. Moreover, a pathological examination of the surgical biopsy demonstrated no esophageal cancer. On the basis of the clinical course of the disease, we strongly suspected a breakthrough of L. lactis and $S$. nepalensis through the ruptured esophagus into the bloodstream. To prove this relationship, we obtained permission from the patient's son to analyze samples of the food products his father consumed, including cheese, dry-cured ham, sauerkraut, pizza margherita, bianchetti (pasta with boiled young sardines), and red and white wine. We cultured samples from these products on blood agar medium; colonies of L. lactis, confirmed by MALDI-TOF mass spectrometry, were derived from cheese samples (Figure).

Approximately 20 cases of L. lactis bacteremia have been reported (1), mostly in immunosuppressed patients with malignancy including leukemia, diabetes, or impaired skin barrier function due to central venous catheter. Several entry routes to the bloodstream have been hypothesized, including the digestive tract or the skin in catheter-related bloodstream infections, or as a result of microbial substitution due to glycopeptide administration; however, no entry point has been definitively identified $(1,2,7,8)$. In addition, $L$. lactis bacteremia caused by gastrointestinal tract perforation had not been reported. We concluded that L. lactis colonized cheese and entered the bloodstream through a perforation of the lower esophagus, and we were able to demonstrate that L. lactis can enter the bloodstream through a rupture of the digestive tract. Based on our findings, we may advise screening for gastrointestinal diseases, such as ulcer, perforation, and malignancy, in patients with L. lactis bacteremia.

S. nepalensis has not previously been reported as a human pathogen, nor has its pathogenicity been described. Because the results of the food sample cultures identified other coagulase-negative Staphylococci bacteria (S. equorum and S. xylosus), rather than $S$. nepalensis, from the dry-cured ham colonies, we could not conclusively demonstrate the entry of $S$. nepalensis to the bloodstream. Moreover, contamination with $S$. nepalensis was possible; only 1 anaerobic bottle of blood culture taken at admission was positive. However, because S. nepalensis is not normally found in the human microbial flora but is a part of the predominant flora in dry-cured ham together with S. equorum and S. xylosus (4), we suspect that the $S$. nepalensis bacteremia diagnosis was correct.

In conclusion, we demonstrate the point of entry for L. lactis into the human bloodstream and show results implying that L. lactis can be a pathogen of bacteremia, as previous reports have shown $(1,2,7,8)$. Our report is also a suspected case of $S$. nepalensis bacteremia; further investigation is needed for confirmation. 


\section{About the Author}

Dr. Hosoya was a junior resident of the Department of Disease Control and Prevention Center, National Center for Global Health and Medicine, Tokyo, Japan at the time of this work. He is an obstetrician-gynecologist with the National Center for Global Health and Medicine. His primary research interest is infectious disease in obstetrics and gynecology.

\section{References}

1. Matsuda K, Koya J, Toyama K, Ikeda M, Arai S, Nakamura F, et al. A therapeutic benefit of daptomycin against glycopeptide-resistant gram-positive cocci bloodstream infections under neutropenia. J Infect Chemother. 2017;23:788-90. https:// doi.org/10.1016/j.jiac.2017.06.010

2. Yang C, Wang D, Zhou Q, Xu J. Bacteremia due to vancomycin-resistant Leuconostoc lactis in a patient with pneumonia and abdominal infection. Am J Med Sci. 2015;349:282-3. https://doi.org/10.1097/ MAJ.0000000000000380

3. Nováková D, Pantůcek R, Petrás P, Koukalová D, Sedlácek I. Occurrence of Staphylococcus nepalensis strains in different sources including human clinical material. FEMS Microbiol Lett. 2006;263:163-8. https://doi.org/10.1111/ j.1574-6968.2006.00408.x

4. Fulladosa E, Garriga M, Martín B, Guàrdia MD, García-Regueiro JA, Arnau J. Volatile profile and microbiological characterization of hollow defect in dry-cured ham. Meat Sci. 2010;86:801-7. https:/ / doi.org/ 10.1016/j.meatsci.2010.06.025

5. Fukami K, Satomi M, Funatsu Y, Kawasaki K, Watabe S. Characterization and distribution of Staphylococcus sp. implicated for improvement of fish sauce odor. Fish Sci. 2004; 70:916-23. https://doi.org/10.1111/j.1444-2906.2004.00887.x

6. Clinical and Laboratory Standards Institute. Methods for antimicrobial dilution and disk susceptibility testing of infrequently isolated or fastidious bacteria. 3rd ed. CLSI guideline M45. Wayne (PA): The Institute; 2016.

7. Lee MR, Huang YT, Lee PI, Liao CH, Lai CC, Lee LN, et al. Healthcare-associated bacteremia caused by Leuconostoc species at a university hospital in Taiwan between 1995 and 2008. J Hosp Infect. 2011;78:45-9. https:/ / doi.org/10.1016/ j.jhin.2010.11.014

8. Patel T, Molloy A, Smith R, Balakrishnan I. Successful treatment of Leuconostoc bacteremia in a neutropenic patient with tigecycline. Infect Dis Rep. 2012;4:e31. https:/ / doi.org/10.4081/idr.2012.e31

Address for correspondence: Satoshi Kutsuna, Department of Disease Control and Prevention Center, National Center for Global Health and Medicine, 1-21-1, Toyama, Shinjuku-ku, Tokyo 162-8655, Japan; email: skutsuna@hosp.ncgm.go.jp

\section{Latent Tuberculosis Screening Using Electronic Health Record Data}

\author{
Jeffrey D. Jenks, Richard S. Garfein, Wenhong Zhu, \\ Michael Hogarth
}

Author affiliation: University of California, La Jolla, California, USA

DOI: https://doi.org/10.3201/eid2609.191391

Screening for latent tuberculosis infection is recommended for foreign-born persons in the United States. We used proxy data from electronic health records to determine that $17.5 \%$ of foreign-born outpatients attending the UC San Diego Health clinic (San Diego, CA, USA) underwent screening. Ending the global tuberculosis epidemic requires improved screening.

7 he World Health Organization End TB Strategy
aims to end the global tuberculosis (TB) epidemic by 2035 (1). The US Preventive Service Task Force (2) and Centers for Disease Control and Prevention (3) recommend screening for latent tuberculosis infection (LTBI) in populations at increased risk for infection or progression to TB disease, including foreignborn persons and former residents of countries with increased TB prevalence. Seventy-four percent of active TB cases in San Diego County, California, USA, occur among foreign-born persons, most of whom are from the Philippines, Vietnam, and Mexico; $80 \%$ result from reactivated LTBI (5). Therefore, TB elimination in the United States requires better diagnosis and treatment of LTBI, especially in foreign-born persons in areas with a low background prevalence of TB, such as San Diego County. However, the frequency of screening for LTBI in foreign-born persons is unknown.

Because medical records often lack information about country of birth, we assessed whether self-reported nationality plus preferred language is a good proxy variable for foreign birth. We used this proxy to determine LTBI screening, prevalence, and treatment rates in foreign-born persons seen at UC San Diego Health (UCSDH) Medical Center in San Diego. We searched the electronic health record (EHR) at UCSDH and validated this search by reviewing a subset of individual EHRs. The University of California San Diego Institutional Review Board approved this study.

We used the clinical data repository module of our EHR, EPIC (https://www.epic.com), to search the records of all patients who accessed care in the outpatient clinic at UCSDH at least once from March 